\title{
Detecting Thermally Induced Spinodal Decomposition with Picosecond Ultrasonics in Cast Austenitic Stainless Steels
}

Saleem Al Dajani ${ }^{1}$, Benjamin Dacus ${ }^{2}$, Cody Dennett ${ }^{3}$, M. Grace Burke ${ }^{4}$, Aljazzy Alahmadi ${ }^{2}$, Kudzanai Mukahiwa $^{5}$, Kuba Anglin ${ }^{6}$, James Wall ${ }^{7}$, Thak Sang Byun ${ }^{8}$ and Michael Short ${ }^{2}$

${ }^{1}$ Mesoscale Nuclear Materials Group, Short Lab, NSE, MIT Nuclear Engineering, University of California Berkeley, Cambridge, Massachusetts, United States, ${ }^{2}$ MIT, Cambridge, Massachusetts, United States, ${ }^{3}$ Idaho National Laboratory, Cambridge, Massachusetts, United States, ${ }^{4}$ University of Manchester, Manchester, Lancashire, England, United Kingdom, ${ }^{5}$ Manchester University, Manchester, Lancashire, England, United Kingdom, ${ }^{6}$ University of Santa Cruz, Santa Cruz, California, United States, ${ }^{7}$ Electric Power Research Institute (EPRI), Charlotte, North Carolina, United States, ${ }^{8}$ Oak Ridge National Laboratory, Oak Ridge, Tennessee, United States

Given the existential climate crisis faced by mankind and the world, the lifetime and sustainability of nuclear reactors as a carbon-free source of renewable energy depend on the susceptibility of their structural components to environmental degradation. In particular, critical components for light water reactors (LWRs) evolve over decades in service, losing ductility and toughness due to thermal and irradiation aging. Techniques to monitor their health cannot be easily applied in the field due to their destructive, expensive, or immobile nature. Thus, non-destructive evaluation (NDE) methods are sought to monitor and evaluate the health of major LWR components such as core barrels, steam generator tubes, or primary coolant pipes and are often required by policy, such as NRC policy \#10-CFR-50.65. Here we demonstrate the use of gigahertz, non-contact ultrasonics to gauge the state of cast austenitic stainless steels (CASS), used in some of the largest components in LWR primary systems. We do so by linking changes in their surface acoustic wave (SAW) characteristics using transient grating spectroscopy (TGS) to transmission electron microscopy (TEM)-verified evidence of spinodal decomposition and G-phase precipitation. In this thesis, thermal aging is shown to induce SAW peak splitting in spinodally decomposed CASS alloys, correlated strongly with lowered toughness and decreased ductility. Furthermore, statistical testing on the number of SAW peak splits observed show that the second SAW peak significantly appears more frequently and is significantly different in frequency in comparison to counts and frequencies measured in unaged specimens. The ability of this technique to non-destructively detect microstructural degradation at a distance in a predictive manner in the case of CASS motivates extending gigahertz ultrasonics to detect other LWR material degradation modes as an in-vessel inspection technique, such as reactor pressure vessel (RPV) embrittlement. This allows for the greater use of NDE techniques for confident monitoring of LWR structural material health to 80 years and beyond, saving costs by minimizing structural replacements until needed and maximizing energy production by preventing early decommission until necessary. 


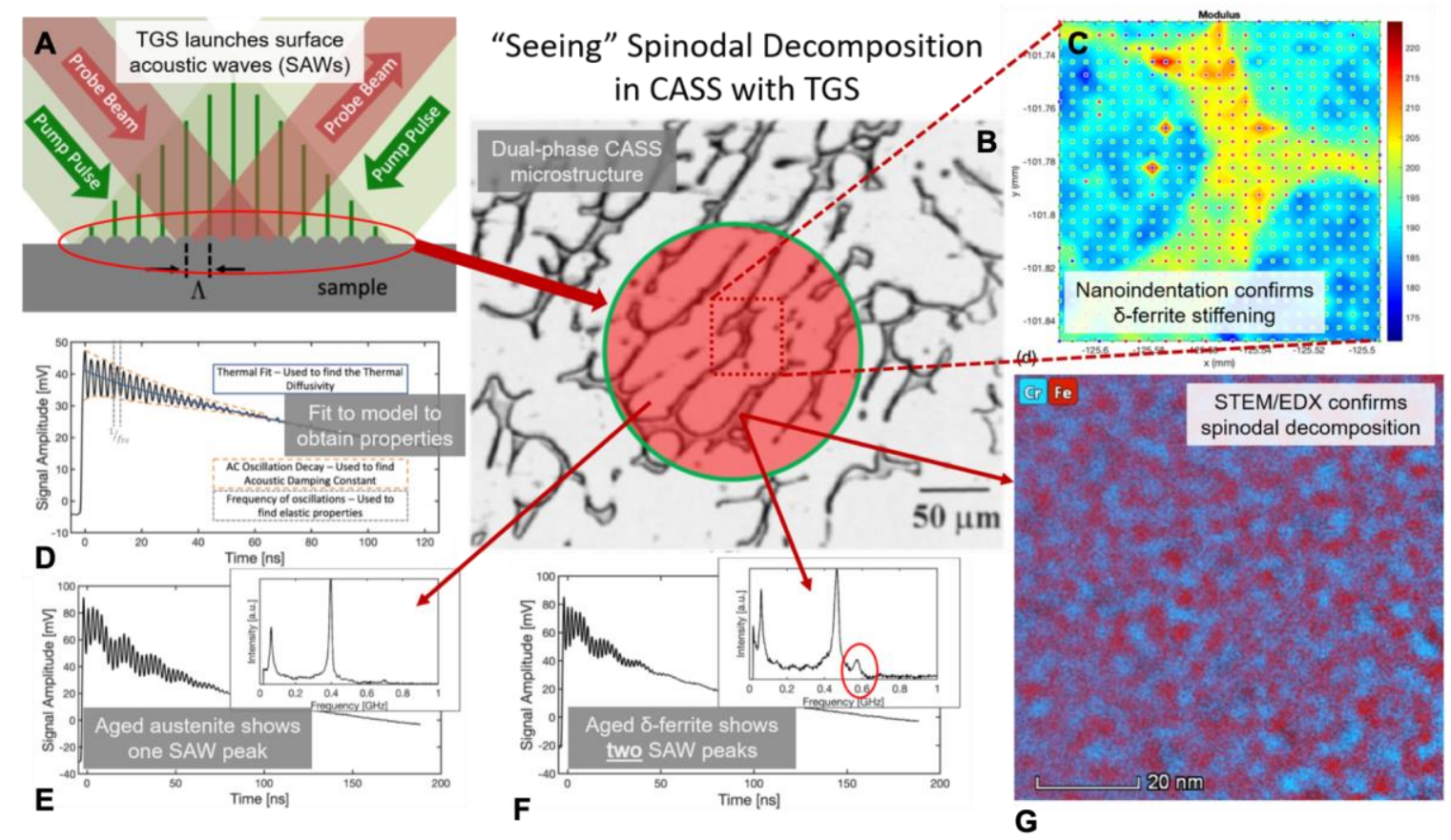

Figure 1. Seeing spinodal decomposition in CASS with TGS (A) Diagram of SAW generation due to pump pulse interference. (Source: S. A. Aldajani et. al. 2019) (B) Microstructure of example CASS alloy, dark gray represents the ferrite phase and light gray represents the austenite phase (Source: M. D. Mathew et. al. 1999) (C) Nanoindentationmap showing $\delta$-ferrite at a higher stiffness than the surrounding austenite (D) Annotated, unaged CF8 CASS TGS signal showing thermal fit (blue), acoustic decay, (red), and frequency of oscillations (dashed). Measurement was taken in vacuum so there is no oscillation due to waves created in air. (Source: S. A. Aldajani et. al. 2019) (E) Unaged CF8 CASS control sample, raw time-domain signal and Fourier transform (inset). The lower frequency peak corresponds to pressure wavea in air, and corresponding oscillations can be seen in the raw signal. (Source: S. A. Aldajani et. al. 2019) (F) CF8 CASS aged at 400C for 10,000 hours, raw time-domain signal and Fourier transform (inset). Fourier frequency on low end of spectrum corresponds to pressure wave in air and corresponding oscillations can be seen in the raw signal. A second peak, attributed to spinodal decomposition in the $\delta$ phase, is seen emerging around $550 \mathrm{MHz}$. (Source: S. A. Aldajani et. al. 2019) (G) Combined Cr and Fe elemental map extracted from a STEM-EDX SI data set showing the classic isotropic spinodally decomposed $\delta$-ferrite. (Source: S. A. Aldajani et. al. 2019)
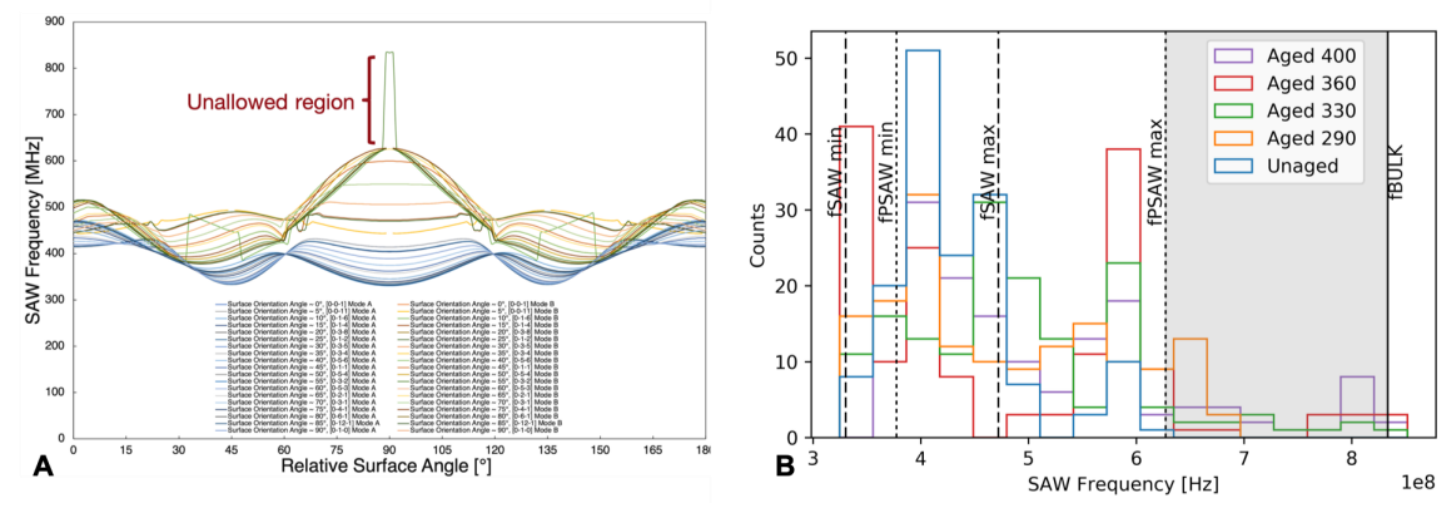
Figure 2. Distinct SAW frequency (second peak) emerges more frequently in spinodally decomposed CASS alloys (A) Predicted acoustic frequencies for a polarization range from 0 to 90 degrees ([ $\left[\begin{array}{lll}0 & 0 & 1\end{array}\right]$ to $\left.\left[\begin{array}{lll}0 & 1 & 0\end{array}\right]\right)$ due to random crystal orientation by mode (as a function of relative surface angle) for pristine (unaged) cast austenitic stainless steel. (B) Distribution of observed SAW frequencies show the emergence of a statistically significant, second Fourier frequency outside the range of predicted frequencies due to random crystal orientation. Shaded region indicates the unallowable range of frequencies due to random crystal orientation, fSAW, min and fSAW, max indicate the expected variation from measuring SAW frequencies of crystals oriented differently, fPSAW, min and FPAW, max indicate the expected variation from measuring PSAW frequencies of crystals oriented differently, and fBULK indicates the allowable fast bulk mode--as predicted by elastic theory.

\section{References}

[1] Danoix, F., P. Auger, and D. Blavette. Microscopy and Microanalysis 10.3 (2004)

[2] Tang, H. T. "Materials Reliability Program: PWR Internals Material Aging Degradation Mechanism Screening and Threshold Values

(MRP-175)." (2005).

[3] Andresen, P. L., et al. Expert Panel Report on Proactive Materials Degradation Assessment (PMDA). 2006.

[4] Busby, Jeremy T., et al. ORNL Report, ORNL/TM-2008/170 (2008)

[5] Johnson, Jeremy A., et al. Journal of Applied Physics 111.2 (2012)

[6] Virkkunen, Iikka, Doug Kull, and Mika Kemppainen. ASME 2012 Pressure Vessels and Piping Conference. American Society of Mechanical Engineers, 2012.

[7] Hofmann, F., et al. Acta Materialia 89 (2015)

[8] Short, Michael P., et al. JOM 67.8 (2015)

[9] Chopra, O. K., and A. S. Rao. Journal of Pressure Vessel Technology 138.4 (2016)

[10] Dennett, Cody A., et al. Physical Review B 94.21 (2016)

[11] Duncan, R. A., et al. Applied Physics Letters 109.15 (2016)

[12] Byun, T. S., et al. JOM 68.2 (2016)

[13] Dennett, Cody A., and Michael P. Short. Applied Physics Letters 110.21 (2017)

[14] Du, Xinpeng, and Ji-Cheng Zhao. Computational Materials 3.1 (2017)

[15] Dennett, C. A., et al. Acta Materialia 145 (2018)

[16] Dennett, Cody A., and Michael P. Short. Journal of Applied Physics 123.21 (2018): 215109.

[17] Short, M. P. Invited Talk, July 2018.

[18] Al Dajani, Saleem et al. Proc. Env. Deg. 2019.

[19] Al Dajani, Saleem et al. MiNES Technical Session, 2019. 